\title{
A Survey on Operator Monotonicity, Operator Convexity, and Operator Means
}

\author{
Pattrawut Chansangiam \\ Department of Mathematics, Faculty of Science, King Mongkut's Institute of Technology Ladkrabang, Bangkok 10520, Thailand \\ Correspondence should be addressed to Pattrawut Chansangiam; pattrawut.c@gmail.com
}

Received 31 July 2015; Accepted 18 October 2015

Academic Editor: Julien Salomon

Copyright (C) 2015 Pattrawut Chansangiam. This is an open access article distributed under the Creative Commons Attribution License, which permits unrestricted use, distribution, and reproduction in any medium, provided the original work is properly cited.

\begin{abstract}
This paper is an expository devoted to an important class of real-valued functions introduced by Löwner, namely, operator monotone functions. This concept is closely related to operator convex/concave functions. Various characterizations for such functions are given from the viewpoint of differential analysis in terms of matrix of divided differences. From the viewpoint of operator inequalities, various characterizations and the relationship between operator monotonicity and operator convexity are given by Hansen and Pedersen. In the viewpoint of measure theory, operator monotone functions on the nonnegative reals admit meaningful integral representations with respect to Borel measures on the unit interval. Furthermore, Kubo-Ando theory asserts the correspondence between operator monotone functions and operator means.
\end{abstract}

\section{Introduction}

A useful and important class of real-valued functions is the class of operator monotone functions. Such functions were introduced by Löwner in a seminal paper [1]. These functions are functions of Hermitian matrices/operators preserving order. In that paper, he established a relationship between operator monotonicity, the positivity of matrix of divided differences, and an important class of analytic functions, namely, Pick functions. This concept is closely related to operator convex/concave functions which was studied afterwards by Kraus in [2]. Operator monotone functions and operator convex/concave functions arise naturally in matrix and operator inequalities (e.g., [3-7]). This is because the theory of inequalities depends heavily on the concepts of monotonicity, convexity, and concavity. One of the most beautiful and important results in operator theory is the so-called LöwnerHeinz inequality (see $[1,8]$ ) which is equivalent to the operator monotonicity of the function $t \mapsto t^{p}$ for $t \geqslant 0$ when $p \in[0,1]$. See more information about operator monotonicity/convexity in $[5$, Chapter V], [9, Section 2], [10, Chapter 4], and [11].

Operator monotone functions have applications in many areas, including functional analysis, mathematical physics, information theory, and electrical engineering; see, for example, [12-15]. This concept plays major roles in the so-called Kubo-Ando theory of operator connections and operator means. This axiomatic theory was introduced in [16] and plays central role in operator inequalities, operator equations, network theory, and quantum information theory. Indeed, there is a one-to-one correspondence between operator monotone functions on the nonnegative reals $\mathbb{R}^{+}$and operator connections. See more information about applications of operator monotone functions to theory of operator means in $[9,10,17-19]$.

In this paper, we survey significant results of operator monotone functions related to operator convexity and operator means. We give various characterizations in several viewpoints. The first viewpoint is differential analysis in terms of matrix of divided differences. In viewpoint of operator inequalities, Hansen and Pedersen provide characterizations and relationship of operator monotonicity and operator convexity/concavity. From measure theory viewpoint, every operator monotone function on the nonnegative reals always occurs as an integral of suitable operator monotone functions with respect to a Borel measure. Such functions form building blocks for arbitrary operator monotone functions on $\mathbb{R}^{+}$. A deep theory of Kubo and Ando states that each operator 
monotone function $f$ on $\mathbb{R}^{+}$corresponds to a unique operator connection. Moreover, if $f(1)=1$, then $f$ is associated with an operator mean.

Here is the outline of the paper. In Section 2, after setting basic notations, we give the definitions and examples of operator monotone/convex functions and provide their characterizations with respect to matrix of divided differences. Section 3 deals with Hansen-Pedersen characterizations of operator monotone/convex functions. The aims of Section 4 are to characterize operator monotone functions on the nonnegative reals in terms of Borel measures and to give some concrete examples. Finally, we establish the correspondence between operator monotone functions and operator means in Section 5.

\section{Operator Monotonicity and Convexity}

Throughout this paper, let $\mathbb{H}$ be a complex Hilbert space. Denote by $B(\mathbb{W})$ the algebra of bounded linear operators on $\mathbb{H}$. The spectrum of an operator $A$ is denoted by $\sigma(A)$. Consider the real vector space $B(\mathbb{H})^{\text {sa }}$ of self-adjoint operators on $\mathbb{W}$ and its positive cone $B(\mathbb{H})^{+}$of positive operators on $\mathbb{H}$. A partial order is naturally equipped on $B(\mathbb{M})^{\text {sa }}$ by defining $A \leqslant B$ if and only if $B-A \in B(\mathbb{U})^{+}$. We write $A>0$ to mean that $A$ is a strictly positive operator, or equivalently, $A \geqslant 0$ and $A$ is invertible. When $\mathbb{H}=\mathbb{C}^{n}$, we identify $B(\mathbb{H})$ with the algebra $\mathbb{M}_{n}$ of $n$-by- $n$ complex matrices. Then $\mathbb{M}_{n}^{+}$is just the cone of $n$-by- $n$ positive semidefinite matrices.

Let $A \in \mathbb{M}_{n}$ be normal. By the spectral resolution of $A$, there exist distinct scalars $\lambda_{1}, \ldots, \lambda_{m} \in \mathbb{C}$ and projections $P_{1}, \ldots, P_{m}$ on $\mathbb{C}^{n}$ such that

$$
\begin{aligned}
A & =\sum_{i=1}^{m} \lambda_{i} P_{i}, \\
P_{i} P_{j} & =0 \quad \text { for } i \neq j, \\
\sum_{i=1}^{m} P_{i} & =I .
\end{aligned}
$$

Moreover, these scalars and projections are uniquely determined. In fact, $\sigma(A)$ is the set $\left\{\lambda_{1}, \ldots, \lambda_{m}\right\}$ (not counting multiplicities) and each $P_{i}$ is the projection onto the eigenspace $\operatorname{ker}\left(A-\lambda_{i} I\right)$. For each function $f: \sigma(A) \rightarrow \mathbb{C}$, we can define the functional calculus for the function $f$ by

$$
f(A)=\sum_{i=1}^{m} f\left(\lambda_{i}\right) P_{i} .
$$

Definition 1. Let $J \subseteq \mathbb{R}$ be an interval. A function $f: J \rightarrow \mathbb{R}$ is said to be as follows:

(i) Matrix monotone of degree $n$ or $n$-monotone if, for every $A, B \in \mathbb{M}_{n}^{\text {sa }}$ with $\sigma(A), \sigma(B) \subseteq J$, it holds that

$$
\begin{gathered}
A \leqslant B \Longrightarrow \\
f(A) \leqslant f(B) .
\end{gathered}
$$

(ii) Operator monotone or matrix monotone if it is $n$ monotone for all $n \in \mathbb{N}$.

(iii) Matrix convex of degree $n$ or $n$-convex if, for every $A, B \in \mathbb{M}_{n}^{\text {sa }}$ with $\sigma(A), \sigma(B) \subseteq J$, it holds that

$$
f(t A+(1-t) B) \leqslant t f(A)+(1-t) f(B) .
$$

(iv) Operator convex if it is $n$-convex for every $n \in \mathbb{N}$.

(v) Matrix concave of degree $n$ or $n$-concave if $-f$ is $n$ convex.

(vi) Operator concave if it is $n$-concave for every $n \in \mathbb{N}$.

Recall that a continuous function $f: J \rightarrow \mathbb{R}$ is convex (concave) if and only if it is midpoint-convex (midpointconcave, resp.). It follows that a continuous function $f: J \rightarrow$ $\mathbb{R}$ is $n$-convex if and only if it is $n$-midpoint convex; that is, the condition (4) holds for $t=1 / 2$. In particular, if $f$ is continuous, then $f$ is operator convex if and only if it is operator midpoint-convex. Similar results are applied for the case of concavity.

Every $n$-monotone function is $(n-1)$-monotone but the converse is false in general. The condition of being 1monotone is the monotone increasing in usual sense. The set of operator monotone functions on the interval $J$ is closed under taking nonnegative linear combinations, pointwise limits, and compositions. The straight line $t \mapsto m t+c$ is operator concave and operator convex on the real line for any $m, c \in \mathbb{R}$. This function is operator monotone if and only if the slope $m$ is nonnegative.

Proposition 2. On $(0, \infty)$, the function $t \mapsto 1 / t$ is operator convex and $t \mapsto-1 / t$ is operator monotone. On $(-\infty, 0)$, the function $t \mapsto 1 / t$ is operator concave and $t \mapsto-1 / t$ is operator monotone.

Proof. If $A \geqslant B>0$, then $A^{-1} \leqslant B^{-1}$ and hence $-A^{-1} \geqslant-B^{-1}$. This shows the operator monotonicity of $t \mapsto-1 / t$ on $(0, \infty)$. The scalar inequality $[(1+t) / 2]^{-1} \leqslant\left(1+t^{-1}\right) / 2$ implies that for every $C>0$

$$
\left(\frac{I+C}{2}\right)^{-1} \leqslant \frac{I+C^{-1}}{2}
$$

For $A, B>0$ in $\mathbb{M}_{n}$, by setting $C=A^{-1 / 2} B A^{-1 / 2}$; we have

$$
\begin{aligned}
\left(\frac{A+B}{2}\right)^{-1} & =\left(\frac{A^{1 / 2}(I+C) A^{1 / 2}}{2}\right)^{-1} \\
& =A^{-1 / 2}\left(\frac{I+C}{2}\right)^{-1} A^{-1 / 2} \\
& \leqslant A^{-1 / 2}\left(\frac{I+C^{-1}}{2}\right) A^{-1 / 2}=\frac{A^{-1}+B^{-1}}{2} .
\end{aligned}
$$

Hence $t \mapsto 1 / t$ is operator convex. For the case $(-\infty, 0)$, consider $-A$ and $-B$ instead of $A$ and $B$.

It follows from this proposition that the function $t \mapsto(c-$ $t)^{-1}$ is operator monotone on $(a, b)$ for any $c \notin(a, b)$. The next 
result is called the Löwner-Heinz inequality. It was first proved by Löwner [1] and also by Heinz [8]. There are many proofs of this fact. The following is due to Pedersen [20].

Theorem 3. For $A \geqslant B$ in $\mathbb{M}_{n}^{+}$and $r \in[0,1]$, one has $A^{r} \geqslant B^{r}$.

Proof. The continuity argument allows us to consider $A \geqslant$ $B>0$. Since $p \mapsto A^{p}$ and $p \mapsto B^{p}$ are continuous, the set

$$
\Omega=\left\{p \in \mathbb{R}: A^{p} \geqslant B^{p}\right\}
$$

is closed. Clearly, $0,1 \in \Omega$. Recall that the set of dyadic numbers in $[0,1]$ is dense in $[0,1]$. Hence, to prove that $[0,1] \subseteq \Omega$, it suffices to show that

$$
\begin{gathered}
p, q \in \Omega \Longrightarrow \\
\frac{p+q}{2} \in \Omega .
\end{gathered}
$$

Suppose $A^{p} \geqslant B^{p}$ and $A^{q} \geqslant B^{q}$. Then $A^{-p / 2} B^{p} A^{-p / 2} \leqslant I$ and

$$
\begin{aligned}
\left\|B^{p / 2} A^{-p / 2}\right\|^{2} & =\left\|\left(B^{p / 2} A^{-p / 2}\right)^{*}\left(B^{p / 2} A^{-p / 2}\right)\right\| \\
& =\left\|A^{-p / 2} B^{p} A^{-p / 2}\right\| \leqslant 1 .
\end{aligned}
$$

Hence, $\left\|B^{p / 2} A^{-p / 2}\right\| \leqslant 1$ and similarly $\left\|B^{q / 2} A^{-q / 2}\right\| \leqslant 1$. Thus

$$
\begin{aligned}
1 & \geqslant\left\|\left(B^{p / 2} A^{-p / 2}\right)^{*}\left(B^{q / 2} A^{-q / 2}\right)\right\| \\
& =\left\|A^{-p / 2} B^{(p+q) / 2} A^{-q / 2}\right\| \geqslant r\left(A^{-p / 2} B^{(p+q) / 2} A^{-q / 2}\right) \\
& =r\left(A^{-(p+q) / 4} B^{(p+q) / 2} A^{-(p+q) / 4}\right) \\
& =\left\|A^{-(p+q) / 4} B^{(p+q) / 2} A^{-(p+q) / 4}\right\| .
\end{aligned}
$$

Here, $r(\cdot)$ denotes the spectral radius. Now,

$$
I \geqslant A^{-(p+q) / 4} B^{(p+q) / 2} A^{-(p+q) / 4}
$$

or $A^{(p+q) / 2} \geqslant B^{(p+q) / 2}$; that is, $(p+q) / 2 \in \Omega$.

Proposition 4. For each $p>1$, the function $t \mapsto t^{p}$ is not operator monotone on $\mathbb{R}^{+}$.

Proof. Consider $A=(3 / 4)\left[\begin{array}{ll}2 & 0 \\ 0 & 1\end{array}\right]$ and $B=(1 / 2)\left[\begin{array}{ll}1 & 1 \\ 1 & 1\end{array}\right]$. Then $A \geqslant B \geqslant 0$. Since $B$ is a projection, for each $p>0$, we have $B^{p}=B$ and

$$
A^{p}-B^{p}=\left[\begin{array}{cc}
\left(\frac{3}{2}\right)^{p}-\frac{1}{2} & -\frac{1}{2} \\
-\frac{1}{2} & \left(\frac{3}{4}\right)^{p}-\frac{1}{2}
\end{array}\right] .
$$

Compute

$$
\operatorname{det}\left(A^{p}-B^{p}\right)=\left(\frac{3}{8}\right)^{p}\left(3^{p}-\frac{2^{p}+4^{p}}{2}\right) .
$$

If $A^{p} \geqslant B^{p}$, we must have $\operatorname{det}\left(A^{p}-B^{p}\right) \geqslant 0$; that is, $\left(2^{p}+\right.$ $\left.4^{p}\right) / 2 \leqslant 3^{p}$, which is false when $p>1$.
Operator monotone functions can be defined in the context of operators acting on a Hilbert space as illustrated in the next theorem. This is why we also call a matrix monotone function an operator monotone function. Note that in this theorem we assume the continuity of $f$ since we need to define the continuous functional calculus of an operator.

Theorem 5. The following statements are equivalent for a continuous function $f:(a, b) \rightarrow \mathbb{R}$ :

(i) $A \leqslant B \Rightarrow f(A) \leqslant f(B)$ for all Hermitian matrices $A, B$ of all orders whose spectra are contained in $(a, b)$;

(ii) $A \leqslant B \Rightarrow f(A) \leqslant f(B)$ for all Hermitian operators $A, B \in B(\mathbb{M})$ whose spectra are contained in $(a, b)$ and for an infinite-dimensional Hilbert space $\mathbb{M}$;

(iii) $A \leqslant B \Rightarrow f(A) \leqslant f(B)$ for all Hermitian operators $A, B \in B(\mathbb{H})$ whose spectra are contained in $(a, b)$ and for all Hilbert spaces $\mathbb{W}$.

Proof. It is obvious that (iii) implies (ii). The implication (ii) $\Rightarrow$ (i) follows by taking an $n$-dimensional subspace.

(i) $\Rightarrow$ (iii). For each finite-dimensional subspace $F$ of $\mathbb{H}$, let $P_{F}$ be the orthogonal projection onto $F$. Suppose that $A \leqslant$ $B$ in $B(\mathbb{U})$ with spectra in $(a, b)$. Consider nets $A_{F}:=P_{F} A P_{F}+$ $c\left(I-P_{F}\right)$ and $B_{F}:=P_{F} B P_{F}+c\left(I-P_{F}\right)$ in $B(\mathbb{H})$, where $c \in(a, b)$ is fixed and a directed set:

$$
\{F: F \text { is a finite-dimensional subspace of } \mathbb{U}\}
$$

with respect to the set inclusion. Since $A_{F} \rightarrow A$ and $B_{F} \rightarrow B$ in the strong-operator topology, we have $f\left(A_{F}\right) \rightarrow f(A)$ and $f\left(B_{F}\right) \rightarrow f(B)$ in the strong-operator topology. Note that

$$
f\left(A_{F}\right)=f\left(P_{F} A P_{F}\right)+f(c)\left(I-P_{F}\right),
$$

where $f\left(P_{F} A P_{F}\right)$ is the functional calculus of $P_{F} A P_{F}$ in $B(F)$. Since $B(F)$ is identified with $\mathbb{M}_{n}$ with $n=\operatorname{dim} F$ and since $P_{F} A P_{F} \leqslant P_{F} B P_{F}$ as elements of $B(F)$, the assumption (i) implies that $f\left(P_{F} A P_{F}\right) \leqslant f\left(P_{F} B P_{F}\right)$ and hence $f\left(A_{F}\right) \leqslant$ $f\left(B_{F}\right)$. By taking the limit in the strong-operator topology, we have $f(A) \leqslant f(B)$.

\section{Differential Analysis of Operator Monotonicity and Convexity}

In this section, we consider topological properties of operator monotone/convex functions. Note that we do not impose a topological assumption on any $n$-monotone/concave function. The following three theorems show that any $n$-monotone/concave function on an open interval is at least continuously differentiable (i.e., $C^{1}$ ) function when $n \geqslant 2$.

Theorem 6. If $f$ is a 2-monotone function on $(a, b)$, then $f$ is $C^{1}$ on $(a, b)$ and $f^{\prime}>0$ unless $f$ is a constant. In particular, every operator monotone function on $(a, b)$ is $C^{1}$.

Proof. The proof is very long and it consists of many details. The original proof is contained in [1]; see also [9, Section 2]. 
Theorem 7. Let $n \geqslant 2$ be an integer. The following statements are equivalent for a function $f:(a, b) \rightarrow \mathbb{R}:$

(1) $f$ is n-monotone on $(a, b)$;

(2) $f$ is $C^{1}$ on $(a, b)$ and $\left[f^{[1]}\left(\lambda_{i}, \lambda_{j}\right)\right]_{i, j=1}^{n} \geqslant 0$ for every choice of $\lambda_{1}<\lambda_{2}<\cdots<\lambda_{n}$ from $(a, b)$.

Here, the 1st divided difference $f^{[1]}$ is defined as follows:

$$
f^{[1]}(x, y):=\frac{f(x)-f(y)}{x-y}, \quad x \neq y,
$$

and $f^{[1]}(x, x):=f^{\prime}(x)$.

Proof. See [1] or [9, Section 2].

Theorem 8. Let $n \geqslant 2$ be an integer. The following statements are equivalent for a function $f:(a, b) \rightarrow \mathbb{R}:$

(1) $f$ is n-convex on $(a, b)$;

(2) $f$ is $C^{2}$ on $(a, b)$ and $\left[f^{[2]}\left(\lambda_{1}, \lambda_{i}, \lambda_{j}\right)\right]_{i, j=1}^{n} \geqslant 0$ for every choice of $\lambda_{1}, \lambda_{2}, \ldots, \lambda_{n}$ from $(a, b)$.

Moreover, if $f$ is operator convex, then $f^{[1]}(\lambda, \cdot)$ is operator monotone for every $\lambda \in(a, b)$. Here, the 2 nd divided difference $f^{[2]}$ is defined to be

$$
f^{[2]}(x, y, z):=\frac{f^{[1]}(x, y)-f^{[1]}(y, z)}{x-z}, \quad x \neq z,
$$

and $f^{[2]}(x, y, x):=f^{\prime \prime}(x)$.

Proof. See [2] or [9, Section 2].

\section{Hansen-Pedersen Characterizations}

In this section, we characterize operator monotone functions in the sense of Hansen-Pedersen [21].

Theorem 9. Let $f:[0, \alpha) \rightarrow \mathbb{R}$ be a function where $0<\alpha \leqslant$ $\infty$. Then the following are equivalent:

(i) $f$ is operator convex on $[0, \alpha)$ and $f(0) \leqslant 0$;

(ii) $f$ is operator convex on $(0, \alpha)$ and $f\left(0^{+}\right) \leqslant f(0) \leqslant$ 0 , where the existence of $f\left(0^{+}\right):=\lim _{t \rightarrow 0^{+}} f(t)$ and $f\left(0^{+}\right) \leqslant f(0)$ are automatic from the operator convexity of $f$ on $(0, \alpha)$;

(iii) $f(t) / t$ is operator monotone on $(0, \alpha)$ and $f\left(0^{+}\right) \leqslant$ $f(0) \leqslant 0$, where the existence of $f\left(0^{+}\right)$and $f\left(0^{+}\right) \leqslant$ $f(0)$ are automatic from the operator monotonicity of $f(t) / t$ on $(0, \alpha)$;

(iv) $f\left(X^{*} A X\right) \leqslant X^{*} f(A) X$ for every $A \in \mathbb{M}_{n}^{\text {sa }}$ with $\sigma(A) \subset$ $[0, \alpha)$, for every $X \in \mathbb{M}_{n}$ with $\|X\| \leqslant 1$ and for every $n \in \mathbb{N}$;

(v) $f\left(X^{*} A X+Y^{*} B Y\right) \leqslant X^{*} f(A) X+Y^{*} f(B) Y$ for every $A, B \in \mathbb{M}_{n}^{s a}$ with $\sigma(A), \sigma(B) \subseteq[0, \alpha)$, for every $X, Y \in$ $\mathbb{M}_{n}$, with $X^{*} X+Y^{*} Y \leqslant I$ and for every $n \in \mathbb{N}$; (vi) $f(P A P) \leqslant P f(A) P$ for every $A \in \mathbb{M}_{n}^{s a}$ with $\sigma(A) \subseteq$ $[0, \alpha)$, for every orthogonal projection $P$ on $\mathbb{C}^{n}$ and for every $n \in \mathbb{N}$.

Proof. See [21] or [9, Theorem 2.5.2].

Theorem 10. If $\alpha=\infty$ and $f(t) \leqslant 0$ for all $t \in[0, \infty)$, then the conditions of Theorem 9 are also equivalent to

(vii) $-f$ being operator monotone on $[0, \infty)$.

Proof. See [21] or [9, Theorem 2.5.3].

Corollary 11. A function on $f:[0, \infty) \rightarrow[0, \infty)$ is operator monotone if and only if $f$ is operator concave.

Proof. This is the equivalence between (i) and (vii) of Theorem 10.

Corollary 12. Consider the following statements for a function $f:(0, \infty) \rightarrow(0, \infty):$

(i) $f$ is operator monotone;

(ii) $t / f(t)$ is operator monotone;

(iii) $f$ is operator concave;

(iv) $1 / f(t)$ is operator convex.

We have (i) $\Leftrightarrow$ (ii) $\Leftrightarrow$ (iii) $\Rightarrow$ (iv).

Proof. (i) $\Rightarrow$ (ii). For any $\epsilon>0, f(t+\epsilon)$ is operator monotone on $[0, \infty)$. Theorem 10 implies that $-f(t+\epsilon) / t$ is operator monotone on $(0, \infty)$. Proposition 2 then implies that

$$
\frac{t}{f(t+\epsilon)}=-\left(-\frac{f(t+\epsilon)}{t}\right)^{-1}
$$

is operator monotone on $(0, \infty)$. Letting $\epsilon \searrow 0$ yields (ii).

(ii) $\Rightarrow$ (i). For any $\epsilon>0,(t+\epsilon) / f(t+\epsilon)$ is operator monotone on $[0, \infty)$. Theorem 10 implies that $-(t+\epsilon) / t f(t+\epsilon)$ is operator monotone on $(0, \infty)$. Proposition 2 then implies that

$$
\frac{t f(t+\epsilon)}{t+\epsilon}=-\left(-\frac{t+\epsilon}{t f(t+\epsilon)}\right)^{-1}
$$

is operator monotone on $(0, \infty)$. Letting $\epsilon \searrow 0$ yields (i).

(i) $\Leftrightarrow$ (iii). By Corollary 11, we have that

$f$ is operator monotone on $(0, \infty)$

$\Longleftrightarrow f(t+\epsilon)$ is operator monotone on $[0, \infty)$

for any $\epsilon>0$

$\Longleftrightarrow f(t+\epsilon)$ is operator concave on $[0, \infty)$

for any $\epsilon>0$

$\Longleftrightarrow f$ is operator concave on $(0, \infty)$.

(iii) $\Leftrightarrow$ (iv). Write $g(t)=1 / f(t)$. Let $A, B>0$ in $\mathbb{M}_{n}$. By (iii),

$$
f\left(\frac{A+B}{2}\right) \geqslant \frac{f(A)+f(B)}{2} .
$$


Then Proposition 2 implies

$$
\begin{aligned}
g\left(\frac{A+B}{2}\right) & =f\left(\frac{A+B}{2}\right)^{-1} \leqslant\left\{\frac{f(A)+f(B)}{2}\right\}^{-1} \\
& \leqslant \frac{f(A)^{-1}+f(B)^{-1}}{2}=\frac{g(A)+g(B)}{2} .
\end{aligned}
$$

Hence $g$ is operator convex.

\section{Example 13. Consider the following:}

(i) For each $p \in[0,1], t^{p}$ is operator concave on $[0, \infty)$.

(ii) The function $f(t)=(t-1) / \log t$ on $[0, \infty)$ where $f(0):=0$ and $f(1):=1$.

(iii) The logarithmic function is operator monotone and operator concave on $(0, \infty)$.

(iv) The function $g(t)=t \log t$ is operator convex on $[0, \infty)$

Proof. (i) It follows from the Löwner-Heinz inequality and Corollary 11. (ii) Use the integral representation $f(t)=$ $\int_{0}^{1} t^{x} d x$ for $t \geqslant 0$. (iii) By (ii), $t / \log (1+t)$ is operator monotone function on $(0, \infty)$. Corollary 12 then implies that $\log (1+$ $t)$ is operator monotone and operator concave on $(0, \infty)$. Now, for each $\epsilon>0, \log (\epsilon+t)=\log \epsilon+\log \left(1+\epsilon^{-1} t\right)$ is operator monotone and operator concave on $(0, \infty)$. Letting $\epsilon \searrow 0$ yields the result. (iv) Since $g$ is continuous on $[0, \infty)$ and $g(t) / t=\log t$ is operator monotone on $(0, \infty)$, we can conclude that $g$ is operator convex on $[0, \infty)$ by Theorem 9.

Example 14. In information theory, the function $f(t)=$ $-t \log t$ is known as the entropy function. In [22], it was shown that this function is operator concave. An analogue notion of the entropy function in quantum mechanics is the entropy of a density matrix (a positive semidefinite matrix with trace 1) or a positive contraction on a Hilbert space. More precisely, for each positive operator $A$ with $\|A\| \leqslant 1$, we define the entropy of $A$ by

$$
S(A)=-A \log A \geqslant 0 .
$$

More concrete examples of operator monotone functions are provided in [23].

\section{Integral Representations of Operator Monotone Functions on the Nonnegative Reals}

In this section, we focus on the class of operator monotone functions from $\mathbb{R}^{+}$to $\mathbb{R}^{+}$, denoted by $\mathrm{OM}\left(\mathbb{R}^{+}\right)$. A reformulation of Löwner's theorem (see [1] or [5, Chapter V]) states that every $f \in \mathrm{OM}\left(\mathbb{R}^{+}\right)$admits an integral representation with respect to Borel measure on the unit interval as follows.

Theorem 15 (see [24]). Given a finite Borel measure $\mu$ on $[0,1]$, the function

$$
f(x)=\int_{[0,1]} 1 !_{t} x d \mu(t), \quad x \geqslant 0
$$

is an operator monotone function from $\mathbb{R}^{+}$to $\mathbb{R}^{+}$. In fact, the map $\mu \mapsto f$ is bijective, affine, and order-preserving.

Thus the functions $x \mapsto 1 !_{t} x$ for $t \in[0,1]$ form building blocks for arbitrary operator monotone functions on $\mathbb{R}^{+}$. The measure $\mu$ in the previous theorem is called the associated measure of the operator monotone function $f$. Moreover, a function $f \in \mathrm{OM}\left(\mathbb{R}^{+}\right)$is normalized (in the sense that $f(1)=1$ ) if and only if $\mu$ is a probability measure [24]. This means that every normalized operator monotone function on $\mathbb{R}^{+}$can be viewed as an average of the special operator monotone functions $x \mapsto 1 !_{t} x$ for $t \in[0,1]$. The functions $x \mapsto 1 !_{t} x$ for $t \in[0,1]$ are extreme points of the convex set of normalized operator monotone functions from $\mathbb{R}^{+}$to $\mathbb{R}^{+}$.

Example 16 (typical examples of "singularly discrete" operator monotone functions). The operator monotone function $x \mapsto$ $1 !_{t} x$ corresponds to the Dirac measure $\delta_{t}$ at $t \in[0,1]$. In particular, the operator monotone functions $x \mapsto 1$ and $x \mapsto x$ correspond to the measures $\delta_{0}$ and $\delta_{1}$, respectively. By affinity of the map $\mu \mapsto f$, the measure $\sum_{i=1}^{n} a_{i} \delta_{t_{i}}$, where $t_{i} \in[0,1]$ and $a_{i} \geqslant 0$, is associated to the function $x \mapsto$ $\sum_{i=1}^{n} a_{i}\left(1 !_{t_{i}} x\right)$.

Example 17. The following examples illustrate the associated measures of "absolutely continuous" operator monotone functions; see [24] for details of proofs.

(1) For each $0<\alpha<1$, the associated measure of the operator monotone function $x^{\alpha}$ is given by

$$
d \mu(t)=\frac{\sin \alpha \pi}{\pi} \cdot \frac{1}{t^{1-\alpha}(1-t)^{\alpha}} d t .
$$

(2) The associated measure of $f(x)=(x-1) / \log x$ is $d \mu(t)=g(t) d t$ where density function $g$ given by

$$
g(t)=\frac{1}{t(1-t)\left(\pi^{2}+\log ^{2}(t /(1-t))\right)} .
$$

(3) Consider the operator monotone function

$$
x \longmapsto \frac{1}{f(1 / x)}=\frac{x}{x-1} \log x .
$$

This function has Lebesgue measure as the associated measure, equivalently; we have the integral representation

$$
\frac{x}{x-1} \log x=\int_{[0,1]} 1 !_{t} x d t
$$

If $f \in \mathrm{OM}\left(\mathbb{R}^{+}\right)$has $\mu$ as the associated measure, then the transpose of $f$, defined by $x \mapsto x f(1 / x)$, also belongs to $\mathrm{OM}\left(\mathbb{R}^{+}\right)$and has $\mu \Theta$ as its associated measure. Here, $\Theta$ : $[0,1] \rightarrow[0,1]$ is a homeomorphism defined by $t \mapsto 1-t$.

We say that $f$ is symmetric if it coincides with its transpose. A Borel measure $\mu$ on $[0,1]$ is said to be symmetric if $\mu$ is invariant under $\Theta$; that is, $\mu \Theta=\mu$. 
Corollary 18 (see [24]). There is a one-to-one correspondence between symmetric operator monotone function from $\mathbb{R}^{+}$to $\mathbb{R}^{+}$ and finite symmetric Borel measures via the integral representation:

$$
f(x)=\frac{1}{2} \int_{[0,1]}\left(1 !_{t} x\right)+\left(x !_{t} 1\right) d \mu(t), \quad x \geqslant 0 .
$$

In particular, an operator monotone function on $\mathbb{R}^{+}$is symmetric if and only if its associated measure is symmetric.

It follows that there is a one-to-one correspondence between normalized symmetric operator monotone functions on $\mathbb{R}^{+}$and probability symmetric Borel measures on the unit interval via the integral representation (29).

The integral representation (24) also has advantages in treating decompositions of operator monotone functions (see [24]). It turns out that every function $f \in \mathrm{OM}\left(\mathbb{R}^{+}\right)$can be expressed as

$$
f=f_{\mathrm{ac}}+f_{\mathrm{sd}}+f_{\mathrm{sc}}
$$

where $f_{\text {ac }}, f_{\text {sd }}$, and $f_{\text {sc }}$ also belong to the class $\mathrm{OM}\left(\mathbb{R}^{+}\right)$. The "singularly discrete part" $f_{\text {sd }}$ is a countable sum of $x \mapsto 1 !_{t} x$ for $t \in[0,1]$ with nonnegative coefficients. The "absolutely continuous part" $f_{\mathrm{ac}}$ has an integral representation with respect to Lebesgue measure $m$ on $[0,1]$. The "singularly continuous part" $f_{\mathrm{sc}}$ has an integral representation with respect to a continuous measure mutually singular to $m$.

\section{Operator Monotone Functions and Operator Means}

This section explains the one-to-one correspondence between operator monotone functions on $\mathbb{R}^{+}$and operator means.

An axiomatic of operator means was investigated by Kubo and Ando [16]. Recall that an operator connection is a binary operation $\sigma$ on $B(\mathbb{H})^{+}$such that for all positive operators $A, B, C, D$ :

(M1) monotonicity: $A \leqslant C, B \leqslant D \Rightarrow A \sigma B \leqslant C \sigma D$;

(M2) transformer inequality: $C(A \sigma B) C \leqslant(C A C) \sigma(C B C)$;

(M3) continuity from above: for $A_{n}, B_{n} \in B(\mathbb{H})^{+}$, if $A_{n} \downarrow A$ and $B_{n} \downarrow B$, then $A_{n} \sigma B_{n} \downarrow A \sigma B$. Here, $X_{n} \downarrow X$ indicates that $\left(X_{n}\right)$ is a decreasing sequence converging strongly to $X$.

An operator mean is an operator connection $\sigma$ with property that $A \sigma A=A$ for all $A \geqslant 0$, or, equivalently, $I \sigma I=0$.

From the monotonicity (M1) and the continuity (M3), every operator connection is uniquely determined on the set of strictly positive operators. Indeed, once an operator connection $\sigma$ is defined for any $A, B>0$, we have

$$
A \sigma B=\lim _{\epsilon \downarrow 0}(A+\epsilon I) \sigma(B+\epsilon I) ;
$$

here the limit is taken in the strong-operator topology.

A major core of Kubo-Ando theory is the one-to-one correspondence between operator connections and operator monotone functions.
Theorem 19 (see [16, Theorem 3.4]). Given an operator connection $\sigma$, there is a unique operator monotone function $f: \mathbb{R}^{+} \rightarrow \mathbb{R}^{+}$such that

$$
f(A)=I \sigma A, \quad A \geqslant 0 .
$$

In fact, the map $\sigma \mapsto f$ is a bijection. Moreover, $\sigma$ is a mean if and only if $\mu$ is a probability measure.

The function $f$ in this theorem is called the representing function of the operator connection $\sigma$. It turns out that, for any $A, B>0$,

$$
A \sigma B=A^{1 / 2} f\left(A^{-1 / 2} B A^{-1 / 2}\right) A^{1 / 2} .
$$

From the integral representation of operator monotone functions (24), every operator connection admits the integral representation

$$
A \sigma B=\int_{0}^{1} A !_{t} B d \mu(t), \quad A, B \geqslant 0 .
$$

Since the weighted harmonic means are jointly concave, it follows that every operator connection is jointly concave.

Example 20. The function $f(x)=2 x /(1+x)$ is operator monotone on $\mathbb{R}^{+}$according to Corollary 12 . This function gives rise to the harmonic mean:

$$
A ! B=2\left(A^{-1}+B^{-1}\right)^{-1}, \quad A, B>0 .
$$

Moreover, $A ! B$ is the largest positive operator $X$ such that

$$
\left[\begin{array}{cc}
2 A & 0 \\
0 & 2 B
\end{array}\right] \geqslant\left[\begin{array}{ll}
X & X \\
X & X
\end{array}\right]
$$

(see [3, Theorem I.3]).

Example 21. For each $\alpha \in(0,1)$, the function $g_{\alpha}(x)=x^{\alpha}$ is operator monotone on $\mathbb{R}^{+}$by the Löwner-Heinz inequality (Theorem 3). Each $g_{\alpha}$ corresponds to the $\alpha$-weighted geometric mean:

$$
A \#_{\alpha} B=A^{1 / 2}\left(A^{-1 / 2} B A^{-1 / 2}\right)^{\alpha} A^{1 / 2}, \quad A, B>0 .
$$

Let us have a close look for the geometric mean $\#_{1 / 2}$, denoted briefly by \#. In the literature, there are various characterizations of the geometric mean. This mean was firstly defined by Pusz and Woronowicz [25]:

$$
\begin{aligned}
& A \# B=\max \{T \geqslant 0:|\langle T x, y\rangle| \\
& \left.\quad \leqslant\left\|A^{1 / 2} x\right\|\left\|B^{1 / 2} y\right\| \forall x, y \in \mathbb{Q}\right\}, \quad A, B \geqslant 0 .
\end{aligned}
$$

This definition coincides with the following formula given by Ando [3]:

$$
A \# B=A^{1 / 2}\left(A^{-1 / 2} B A^{-1 / 2}\right)^{1 / 2} A^{1 / 2}, \quad A, B>0 .
$$


Alternatively, the geometric mean of $A, B>0$ is the common limit of the following iterative process (see [26]):

$$
\begin{aligned}
& A_{0}=A, \\
& B_{0}=B, \\
& A_{n}=A_{n-1} \nabla B_{n-1}, \\
& B_{n}=A_{n-1} ! B_{n-1} .
\end{aligned}
$$

It was also pointed out in [14] that the geometric mean of $A, B>0$ is the unique positive solution to the Riccati equation:

$$
X A^{-1} X=B
$$

Moreover, the geometric mean $A \# B$ is the largest self-adjoint operator $X$ for which

$$
\left[\begin{array}{ll}
A & X \\
X & B
\end{array}\right] \geqslant 0
$$

(see [3, Theorem I.2]). See more information of the geometric mean in [6, Section 4.1] and [10, Section 5.1].

Example 22. For each $p \in[-1,1]$ and $\alpha \in[0,1]$, consider the operator monotone function (see e.g., [5]):

$$
f_{p, \alpha}(x)=\left(1-\alpha+\alpha x^{p}\right)^{1 / p}, \quad x \geqslant 0 .
$$

When $p=0$, it is understood that we take limit as $p$ tends to 0 and, by L'Hôspital's rule,

$$
f_{0, \alpha}(x)=x^{\alpha} \text {. }
$$

Each function $f_{p, \alpha}$ gives rise to a unique operator mean, namely, the quasiarithmetic power mean $\#_{p, \alpha}$ with exponent $p$ and weight $\alpha$ as follows:

$$
A \#_{p, \alpha} B=\left[(1-\alpha) A^{p}+\alpha B^{p}\right]^{1 / p}, \quad A, B \geqslant 0 .
$$

The family of quasiarithmetic power means includes the weighted arithmetic means (the case $p=1$ ), the weighted harmonic means (the case $p=-1$ ), and the weighted geometric means (the case $p=0$ ) as special cases.

Example 23. Let $r \in[-1,1]$ and consider the operator monotone function (see [27])

$$
g_{r}(x)=\left(\frac{3 r-1}{3 r+1}\right) \frac{x^{(3 r+1) / 2}-1}{x^{(3 r-1) / 2}-1}, \quad x \geqslant 0 .
$$

Since each $g_{r}$ is symmetric and normalized, it associates to a unique symmetric operator mean. Note that the family of these means include the arithmetic mean, the geometric mean, and the harmonic mean. The cases $r=1 / 3$ and $r=$ $-1 / 3$ are known as the logarithmic mean and its dual.

Example 24. For each $\alpha \in[0,1 / 2]$, the function

$$
h_{\alpha}(x)=\frac{x^{\alpha}+x^{1-\alpha}}{2}
$$

belongs to $\mathrm{OM}\left(\mathbb{R}^{+}\right)$. Each $h_{\alpha}$ gives rise to an operator mean, called the Heinz mean. See more information in [28].
Example 25. For each $p \in[-2,2]$, consider the operator monotone function

$$
f_{p}(x)=\left(\frac{p(x-1)}{x^{p}-1}\right)^{1 /(1-p)}
$$

where the case $p=1$ is understood as

$$
f_{1}(x)=\lim _{p \rightarrow 1} f_{p}(x)=\frac{1}{e} x^{x /(x-1)} .
$$

The case $p=1$ gives rise to the operator mean, called the identric mean. The case $p=0$ is associated to the logarithmic mean.

Example 26. The function $f(t)=\log t$ is operator monotone on $(0, \infty)$; see, for example, [5]. Fujii and Kamei [29] introduced the relative operator entropy to be the operator connection associated with this function by (33) as follows:

$$
S(A \mid B)=A^{1 / 2} \log \left(A^{-1 / 2} B A^{-1 / 2}\right) A^{1 / 2}, \quad A, B>0 .
$$

Note that $S(I \mid A)=S(A)$ for any $A>0$. The relative operator entropy satisfies attractive entropy-like properties in physical literature (see also [30]).

The relative operator entropy is closely related to the Karcher mean theory. The Karcher mean or the weighted leastsquares mean of the tuple $A=\left(A_{1}, A_{2}, \ldots, A_{n}\right)$ of strictly positive operators with weights $w=\left(w_{1}, w_{2}, \ldots, w_{n}\right)$ of positive real numbers such that $\sum w_{i}=1$ is geometrically defined to be

$$
\arg \min _{X>0} \sum_{i=1}^{n} w_{i} \delta^{2}\left(X, A_{i}\right)
$$

Here, $\delta$ is a Riemannian metric given by

$$
\delta(A, B)=\left\|\log A^{-1 / 2} B A^{-1 / 2}\right\|, \quad A, B>0
$$

It turns out that the Karcher mean (51) is the unique positive definite solution to the equation

$$
\sum_{i=1}^{n} S\left(X \mid A_{i}\right)=0
$$

See more information about Karcher mean theory in [31-34] and references therein.

\section{Conflict of Interests}

The author declares that there is no conflict of interests regarding the publication of this paper.

\section{Acknowledgments}

The author would like to thank referees for valuable suggestions. The author was supported by King Mongkuts Institute of Technology Ladkrabang Research Fund Grant no. KREF045710. 


\section{References}

[1] C. Löwner, “Über monotone matrix funktionen," Mathematische Zeitschrift, vol. 38, pp. 177-216, 1934.

[2] F. Kraus, "Über konvexe Matrixfunktionen," Mathematische Zeitschrift, vol. 41, no. 1, pp. 18-42, 1936.

[3] T. Ando, Topics on Operator Inequalities, Hokkaido University, Sapporo, Japan, 1978.

[4] T. Ando, "Concavity of certain maps on positive definite matrices and applications to Hadamard products," Linear Algebra and its Applications, vol. 26, pp. 203-241, 1979.

[5] R. Bhatia, Matrix Analysis, Springer, New York, NY, USA, 1996.

[6] R. Bhatia, Positive Definite Matrices, Princeton Series in Applied Mathematics, Princeton University Press, Princeton, NJ, USA, 2007.

[7] X. Zhan, Matrix Inequalities, Springer-Verlag, New York, NY, USA, 2002.

[8] E. Heinz, "Beiträge zur Störungstheorie der Spektralzerlegung," Mathematische Annalen, vol. 123, pp. 415-438, 1951.

[9] F. Hiai, "Matrix analysis: matrix monotone functions, matrix means, and majorization," Interdisciplinary Information Sciences, vol. 16, no. 2, pp. 139-248, 2010.

[10] F. Hiai and D. Petz, Introduction to Matrix Analysis and Applications, Springer-Verlag, New Delhi, India, 2014

[11] F. Hiai and K. Yanagi, Hilbert Spaces and Linear Operators, Makino Publishing, 1995.

[12] W. N. Anderson Jr. and G. E. Trapp, "A class of monotone operator functions related to electrical network theory," Linear Algebra and Its Applications, vol. 15, no. 1, pp. 53-67, 1976.

[13] W. N. Anderson Jr., T. D. Morley, and G. E. Trapp, "Positive solutions to $=A-B X^{-1} B$," Linear Algebra and Its Applications, vol. 134, pp. 53-62, 1990.

[14] W. N. Anderson Jr. and G. E. Trapp, "Operator means and electrical networks," in Proceedings of the IEEE International Symposium on Circuits and Systems, pp. 523-527, 1980.

[15] E. P. Wigner and J. von Neumann, "Significance of Löwner's theorem in the quantum theory of collisions," Annals of Mathematics, vol. 59, pp. 418-433, 1954.

[16] F. Kubo and T. Ando, "Means of positive linear operators," Mathematische Annalen, vol. 246, no. 3, pp. 205-224, 1980.

[17] P. Chansangiam and W. Lewkeeratiyutkul, "Characterizations of connections for positive operators," Southeast Asian Bulletin of Mathematics, vol. 37, no. 5, pp. 645-657, 2013.

[18] P. Chansangiam, "Operator means and applications," in Linear Algebra: Theorems and Applications, H. A. Yasser, Ed., chapter 8, InTech, Rijeka, Croatia, 2012.

[19] P. Chansangiam, "Positivity, betweenness, and strictness of operator means," Abstract and Applied Analysis, vol. 2015, Article ID 851568, 5 pages, 2015.

[20] G. K. Pedersen, "Some operator monotone functions," Proceedings of the American Mathematical Society, vol. 36, pp. 309-310, 1972.

[21] F. Hansen and G. K. Pedersen, "Jensen's inequality for operators and Löwner's theorem," Mathematische Annalen, vol. 258, no. 3, pp. 229-241, 1982.

[22] M. Nakamura and H. Umegaki, "A note on the entropy for operator algebras," Proceedings of the Japan Academy, vol. 37, pp. 149-154, 1961.

[23] T. Furuta, "Concrete examples of operator monotone functions obtained by an elementary method without appealing to
Löwner integral representation," Linear Algebra and its Applications, vol. 429, no. 5-6, pp. 972-980, 2008.

[24] P. Chansangiam, "Integral representations anddecompositions of operator monotone functions on the nonnegative reals," http://arxiv.org/abs/1304.7936vl.

[25] W. Pusz and S. L. Woronowicz, "Functional calculus for sesquilinear forms and the purification map," Reports on Mathematical Physics, vol. 8, no. 2, pp. 159-170, 1975.

[26] W. N. Anderson Jr., T. D. Morley, and G. E. Trapp, "Characterization of parallel subtraction," Proceedings of the National Academy of Sciences of the United States of America, vol. 76, no. 8, pp. 3599-3601, 1979.

[27] J. I. Fujii and Y. Seo, "On parametrized operator means dominated by power ones," Scientiae Mathematicae, vol. 1, no. 3, pp. 301-306, 1998.

[28] R. Bhatia, "Interpolating the arithmetic-geometric mean inequality and its operator version," Linear Algebra and its Applications, vol. 413, no. 2-3, pp. 355-363, 2006.

[29] J. Fujii and E. Kamei, "Relative operator entropy in noncommutative information theory," Mathematica Japonica, vol. 34, no. 3, pp. 341-348, 1989.

[30] J. I. Fujii, "Operator means and the relative operator entropy," in Operator Theory and Complex Analysis, vol. 59 of Operator Theory: Advances and Applications, pp. 161-172, Birkhäuser, Basel, Switzerland, 1992.

[31] J. Lawson and Y. Lim, "Monotonic properties of the least squares mean," Mathematische Annalen, vol. 351, no. 2, pp. 267-279, 2011.

[32] J. Lawson and Y. Lim, "Weighted means and Karcher equations of positive operators," Proceedings of the National Academy of Sciences of the United States of America, vol. 110, no. 39, pp. 15626-15632, 2013.

[33] J. D. Lawson and Y. Lim, "Karcher means and Karcher equations of positive definite operators," Transactions of the American Mathematical Society Series B, vol. 1, pp. 1-22, 2014.

[34] M. Moakher, "A differential geometric approach to the geometric mean of symmetric positive-definite matrices," SIAM Journal on Matrix Analysis and Applications, vol. 26, no. 3, pp. 735-747, 2005. 


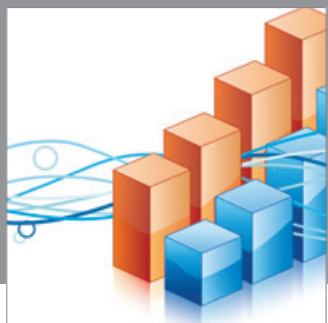

Advances in

Operations Research

mansans

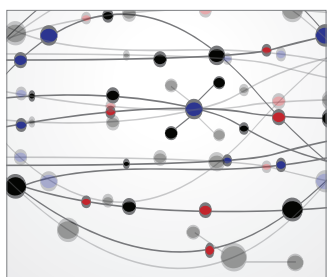

The Scientific World Journal
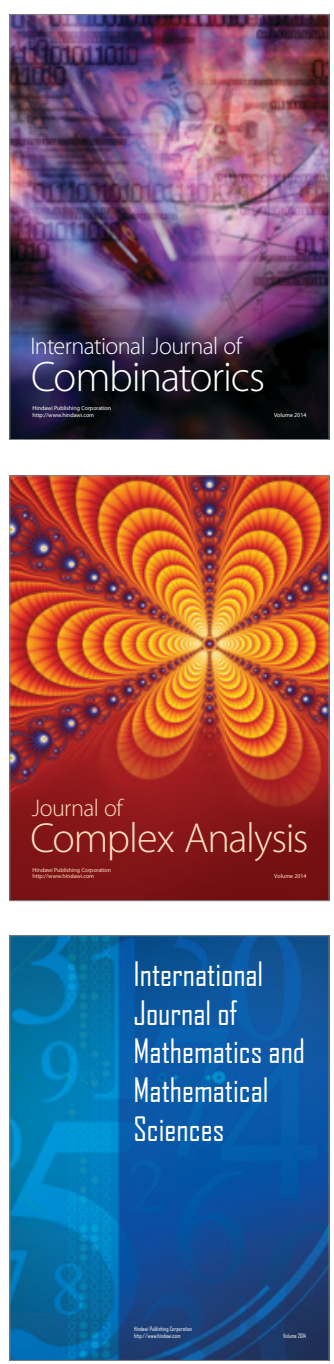
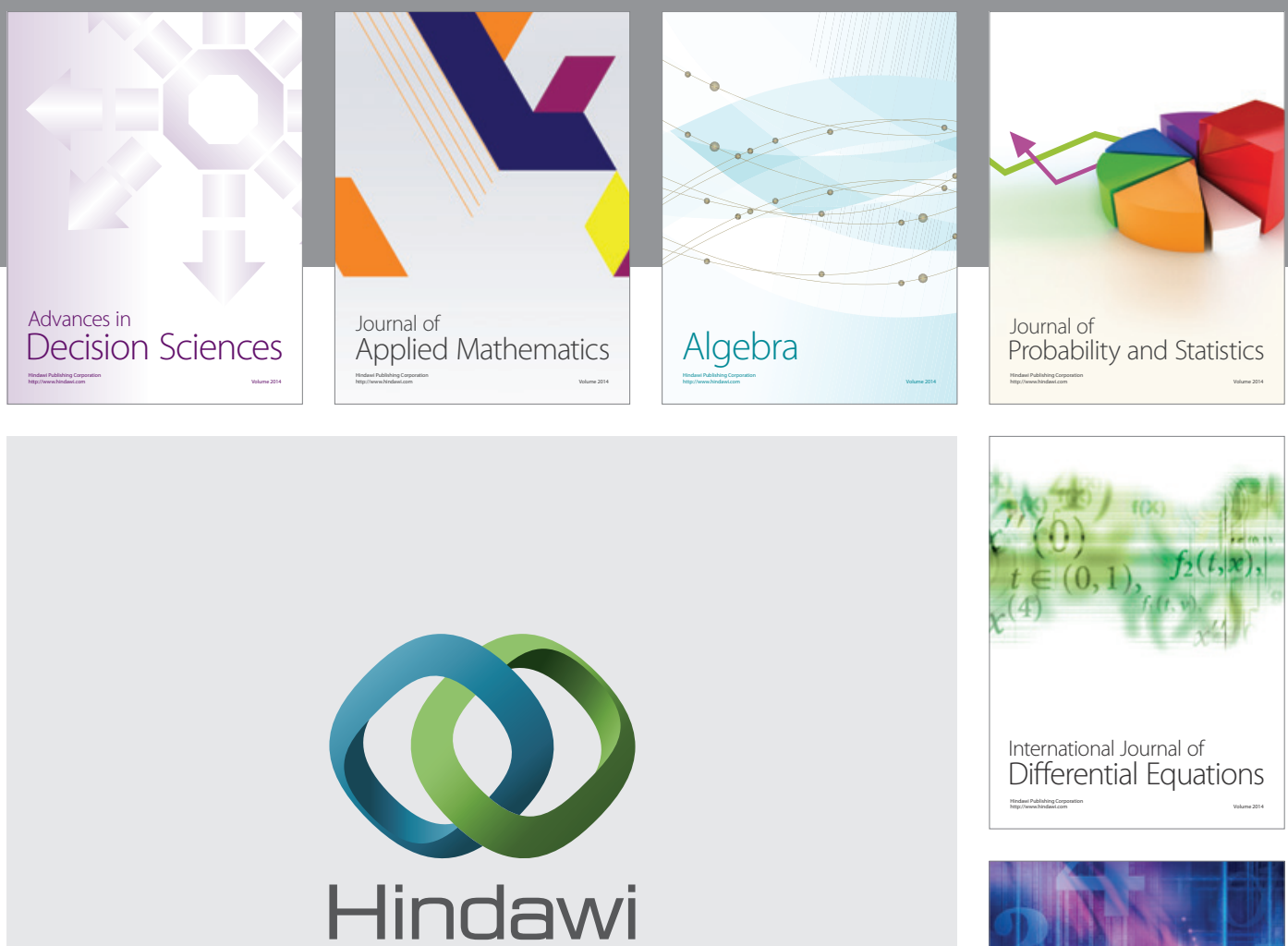

Submit your manuscripts at http://www.hindawi.com
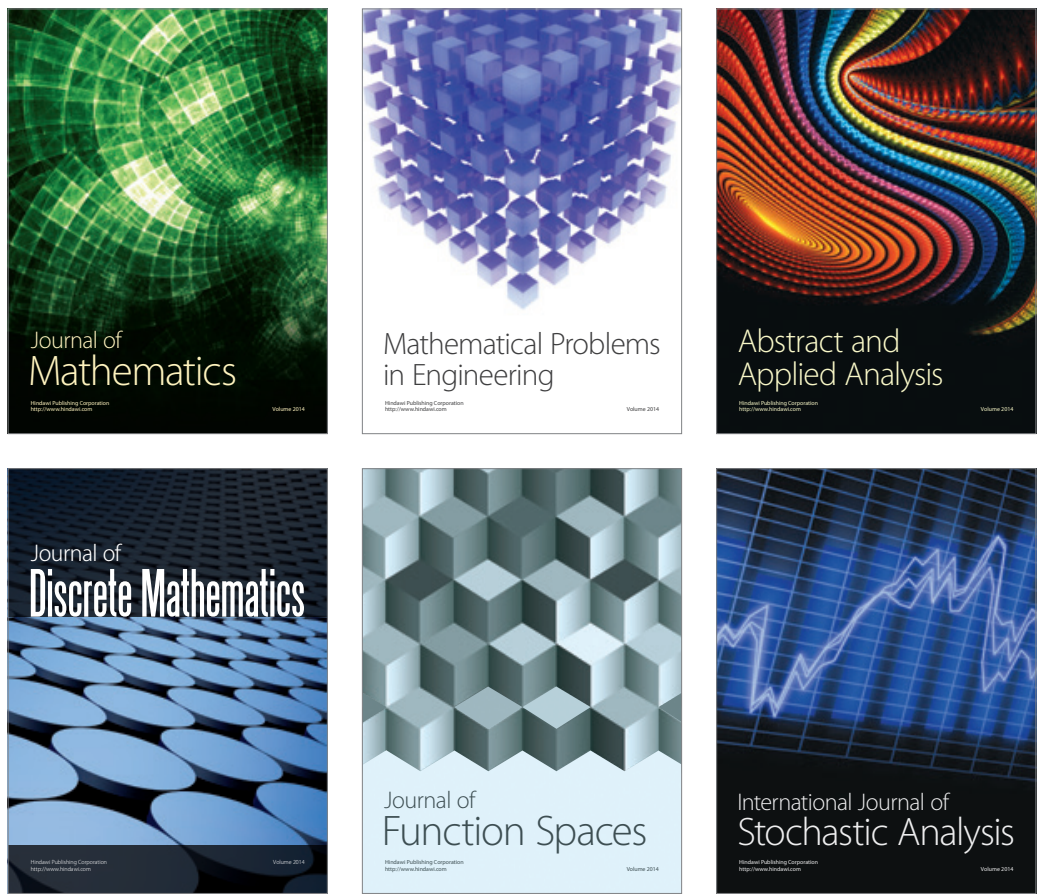

Journal of

Function Spaces

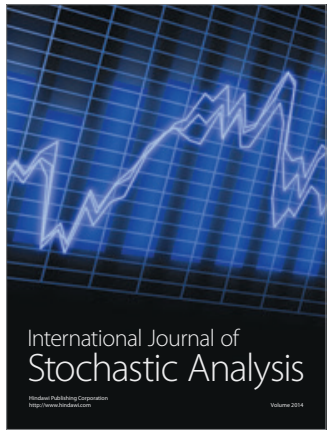

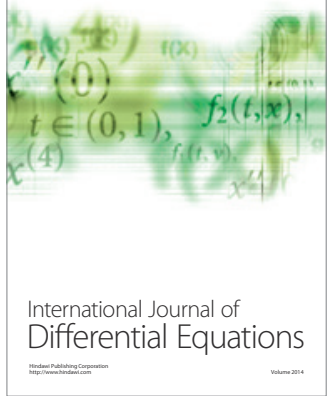
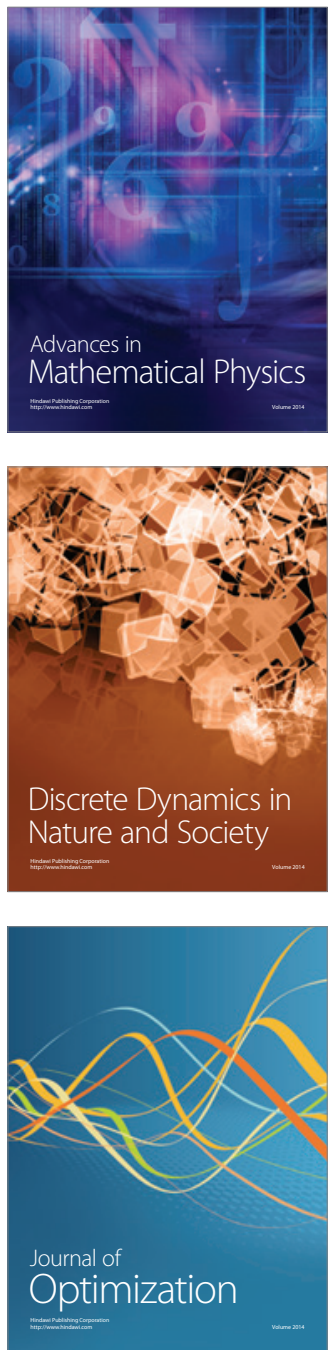